\title{
Genome-wide analysis of the bZIP gene family in Chinese jujube (Ziziphus jujuba Mill.)
}

Yao Zhang ${ }^{1,2+}$, Weilin Gao ${ }^{1,2+}$, Hongtai Li ${ }^{1,2}$, Yongkang Wang ${ }^{3}$, Dengke $\mathrm{Li}^{3}$, Chaoling Xue ${ }^{1,2}$, Zhiguo Liu ${ }^{4}$, Mengjun Liu ${ }^{4}$ and Jin Zhao ${ }^{1,2^{*}}$ (D)

\begin{abstract}
Background: Among several TF families unique to eukaryotes, the basic leucine zipper (bZIP) family is one of the most important. Chinese jujube (Ziziphus jujuba Mill.) is a popular fruit tree species in Asia, and its fruits are rich in sugar, vitamin C and so on. Analysis of the bZIP gene family of jujube has not yet been reported. In this study, ZjbZIPs were identified firstly, their expression patterns were further studied in different tissues and in response to various abiotic and phytoplasma stresses, and their protein-protein interactions were also analyzed.

Results: At the whole genome level, 45 ZjbZIPs were identified and classified into 14 classes. The members of each class of bZIP subfamily contain a specific conserved domain in addition to the core bZIP conserved domain, which may be related to its biological function. Relative Synonymous Codon Usage (RSCU) analysis displayed low values of NTA and NCG codons in ZjbZIPs, which would be beneficial to increase the protein production and also indicated that ZjbZIPs were at a relative high methylation level. The paralogous and orthologous events occurred during the evolutionary process of ZjbZIPs. Thirty-four ZjbZIPs were mapped to but not evenly distributed among 10 pseudo- chromosomes. 30 of ZjbZIP genes showed diverse tissue-specific expression in jujube and wild jujube trees, indicating that these genes may have multiple functions. Some ZjbZIP genes were specifically analyzed and found to play important roles in the early stage of fruit development. Moreover, some ZjbZIPs that respond to phytoplasma invasion and abiotic stress environmental conditions, such as salt and low temperature, were found. Based on homology comparisons, prediction analysis and yeast two-hybrid, a protein interaction network including 42 ZjbZIPs was constructed.
\end{abstract}

Conclusions: The bioinformatics analyses of 45 ZjbZIPs were implemented systematically, and their expression profiles in jujube and wild jujube showed that many genes might play crucial roles during fruit ripening and in the response to phytoplasma and abiotic stresses. The protein interaction networks among ZjbZIPs could provide useful information for further functional studies.

Keywords: ZjbZIPS, Chinese jujube, Tissue-specific expression, Fruit development, Phytoplasma, Abiotic stress, Protein-protein interaction

\footnotetext{
* Correspondence: zhaojinbd@126.com

${ }^{\dagger}$ Yao Zhang and Weilin Gao contributed equally to this work.

${ }^{1}$ College of Life Science, Hebei Agricultural University, Baoding, China

${ }^{2}$ Hebei Key Laboratory of Plant Physiology and Molecular Pathology, Hebei

Agricultural University, Baoding, China

Full list of author information is available at the end of the article
}

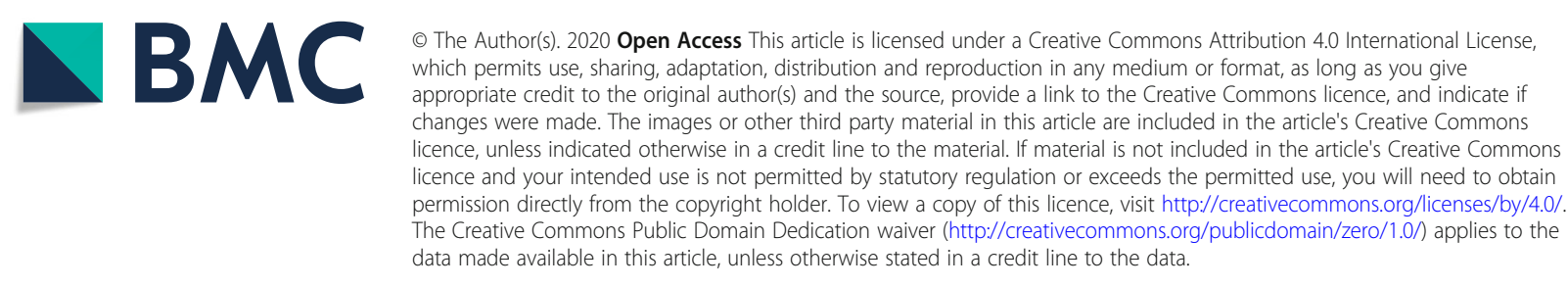




\section{Background}

Transcription factors (TFs) are an important component of regulatory networks. TFs bind to specific promoter sequences to activate or inhibit the expression of target genes [1]. Among several TF families unique to eukaryotes, the basic leucine zipper (bZIP) family is one of the largest and most diverse [2-4], containing two regions with different functions: the basic region and the leucine zipper region [5]. The basic region is highly conserved and consists of approximately 16 amino acid residues with a consistent $\mathrm{N}-\mathrm{X} 7-\mathrm{R} / \mathrm{K}$ motif for nuclear localization and sequentially specific DNA binding, while the leucine zipper region is less conserved and forms a helical structure with dimerization specificity $[1,6]$.

Members of the bZIP family are involved in the regulation of plant resistance under biotic and abiotic stresses [7-9], and play some important roles during plant growth and development processes, such as hormone signal transduction [10], energy metabolism [11], seedling development [12] and flowering [13]. In plant, bZIPs can be combined with cis- acting elements such as G-box (CACGTG), A-box (TACGTA) and abscisic acid (ABA)responsive elements (ABRE) (CCACGTGG) to regulate the expression of downstream genes. In Arabidopsis, ABF1, ABF2, ABF3 and ABF4 could bind to the cis-acting element $A B R E$, and regulate many downstream salt and drought tolerances through the interaction between ABRE and bZIP proteins [14]. OsbZIP1 might enhance resistance to Magnaporthe grisea through salicylic acid (SA), jasmonic acid (JA) and ABA signal transduction pathways [15]. In addition, AtbZIPs also regulated the signal transduction of ABA-related pathways, thereby affecting seed germination and maturity [12].

The TGA (TGACG motif-binding factor) subfamily of bZIPs plays important roles in defense responses against pathogens [16]. As the target of SA signaling, the TGA factors can thus activate and connect the SA pathway with the JA/ET-dependent pathway [17-20]. Moreover, these WRKY transcription factors induced by SA could activate the promoter of pathogenesis-related (PR)-1 and involved in the regulation of the TGA/NPR1 complex $[21,22]$. And they also demonstrated some reverse functions, such as TGA2 suppressed the expression of PR1 whereas TGA6 was able to increase PR1 expression and could induce basal defense [23]. Thus, such interactions will be discovered in more plants when further exploring the function of TGA factors.

Chinese jujube (Ziziphus jujuba Mill.), a member of the Rhamnaceae family, is an important dry fruit and a traditional herbal medicine in Asia [24]. Both Chinese jujube and wild jujube, which are considered ideal fruit trees in arid and semiarid temperate regions, have strong tolerance to biotic and abiotic stress [25, 26]. Although the bZIP gene family has been analyzed in many other plant species such as Arabidopsis [27], peach [28], apple [3] and so on [29, 30], the analysis of bZIP family in jujube has not yet been reported. Based on the functions of bZIPs in other species, we thought the members of bZIP family should have multiple functions on jujube development, defense responses against pathogens and abiotic stress. Thus, the characteristics and expressions of bZIP members in Chinese jujube are identified and analyzed systematically. These results would provide a basis for future studies related to biological functions and the regulatory networks.

\section{Results}

\section{Identification of ZjbZIPs in Chinese jujube}

A total of 45 nonredundant putative bZIP transcripts (Table 1) within the jujube genome sequence were identified. They were named ZjbZIP1 to ZjbZIP45 according to their gene structure and motifs. The ORF length of the ZjbZIPs ranged from $384 \mathrm{bp}$ (ZjbZIP44) to $2205 \mathrm{bp}$ (ZjbZIP11), and they encoded proteins ranging from 127 (ZjbZIP44) to 749 (ZjbZIP11) amino acids (aa) in length, with predicted pIs ranging from 4.65 (ZjbZIP40) to 9.96 (ZjbZIP44) (Table 1) and predicted molecular weights (MWs) of $14.67-82.15 \mathrm{kDa}$. The proteins with an isoelectric point greater than 7 accounted for $53 \%$ of the total number, which means that half of the ZjbZIP proteins were neutral or alkaline, and most of the proteins in the $\mathrm{E}, \mathrm{F}$ and $\mathrm{G}$ subfamilies were weakly acidic.

The average GC content of 45 ZjbZIPs was $46.88 \%$, and the contents of $\mathrm{GC}_{1}, \mathrm{GC}_{2}$ and $\mathrm{GC}_{3}$ were 53.45, 44.63 and $42.56 \%$, respectively. Relative Synonymous Codon Usage (RSCU) analyses will help us to understand the patterns in ZjbZIPs, and the RSCU values greater than 1.5 was defined as high-frequency codons [31]. Among the 64 codons of 45 ZjbZIPs, seven highfrequency codons (AGG 1.80, AGA 1.79, GTT 1.73, GCT 1.69, TTG 1.65, TCT 1.59 and TTT 1.58) were investigated, and most of them were T-ended (Table 2). We also found that most ZjbZIPs prefer ATG as the stop codon. RSCU values of four NCG codons in ZjbZIPs were the lowest (TCG 0.62, CCG 0.61, ACG 0.45 , GCG 0.36) (Table 2), suggesting that ZjbZIPs were at a relative high methylation level [32]. Meanwhile, RSCU values of four NTA codons also displayed a lower level (ATA 0.75, TTA 0.73, GTA 0.66, CTA 0.55), which was beneficial to increase the protein production by inhibiting mRNA degradation [33].

\section{Phylogenetic tree construction and conserved motifs of ZjbZIPs}

Compared with bZIPs in Arabidopsis, ZjbZIPs were also divided into 10 subfamilies (A-I, S). In addition, we defined six newly discovered ZjbZIPs as four subfamilies of J, K, L and M (Fig. 1). The classification result was 
Table 1 The information of bZIP gene family in Chinese jujube

\begin{tabular}{|c|c|c|c|c|c|c|c|c|}
\hline & NCBI accession & Group & Chr. & Size (aa) & $\mathrm{MW}(\mathrm{D})$ & $\mathrm{PI}$ & ORF (bp) & Exon number \\
\hline ZjbZIP1 & XM_016043261.2 & $A$ & 12 & 436 & $47,951.52$ & 8.47 & 1311 & 4 \\
\hline ZjbZIP2 & XM_016043231.2 & A & 12 & 270 & $30,201.81$ & 8.89 & 813 & 3 \\
\hline ZjbZIP3 & XM_016020783.2 & A & 3 & 321 & $35,584.09$ & 8.5 & 966 & 3 \\
\hline ZjbZIP4 & XM_016030823.2 & A & 6 & 299 & $32,803.72$ & 7.79 & 900 & 4 \\
\hline ZjbZIP5 & XM_016030951.2 & A & 6 & 313 & $33,876.96$ & 9.09 & 942 & 4 \\
\hline ZjbZIP6 & XM_016032146.2 & A & 7 & 325 & $35,538.02$ & 5.51 & 978 & 3 \\
\hline ZjbZIP7 & XM_016017070.2 & A & 2 & 397 & $43,940.78$ & 7.09 & 1194 & 4 \\
\hline ZjbZIP8 & XM_016034266.2 & A & 8 & 265 & $29,175.47$ & 9.26 & 798 & 3 \\
\hline ZjbZIP9 & XM_016037430.2 & A & 9 & 434 & $46,121.77$ & 9.85 & 1305 & 4 \\
\hline ZjbZIP10 & XM_025074624.1 & A & 6 & 425 & $45,632.07$ & 9.21 & 1278 & 4 \\
\hline ZjbZIP11 & XM_016042475.2 & B & 12 & 749 & $82,145.02$ & 7.07 & 2250 & 2 \\
\hline ZjbZIP12 & XM_016036011.2 & $C$ & 9 & 449 & $48,518.82$ & 6.2 & 1350 & 6 \\
\hline ZjbZIP13 & XM_016019943.2 & $C$ & 2 & 437 & 47,207 & 5.91 & 1314 & 6 \\
\hline ZjbZIP14 & XM_016017317.2 & D & 2 & 515 & $57,096.85$ & 7.37 & 1548 & 12 \\
\hline ZjbZIP15 & XM_016010890.2 & $\mathrm{D}$ & UN & 467 & $51,490.68$ & 7.09 & 1404 & 11 \\
\hline ZjbZIP16 & XM_016040197.2 & $\mathrm{D}$ & 10 & 468 & $51,929.31$ & 6.07 & 1407 & 11 \\
\hline ZjbZIP17 & XM_016035424.2 & $\mathrm{D}$ & 8 & 363 & $41,012.5$ & 7.07 & 1092 & 8 \\
\hline ZjbZIP18 & XM_025069094.1 & $D$ & UN & 488 & $47,387.8$ & 7.32 & 1269 & 11 \\
\hline ZjbZIP19 & XM_025076058.1 & D & 7 & 348 & $39,084.03$ & 6.09 & 1047 & 8 \\
\hline ZjbZIP20 & XM_016039294.2 & D & 10 & 396 & $44,113.28$ & 6.63 & 1191 & 9 \\
\hline ZjbZIP21 & XM_016043667.2 & $E$ & 12 & 343 & $38,339.63$ & 6.25 & 1032 & 4 \\
\hline ZjbZIP22 & XM_016037719.2 & $\mathrm{F}$ & 9 & 272 & $29,467.89$ & 6.21 & 819 & 2 \\
\hline ZjbZIP23 & XM_016026428.2 & $\mathrm{F}$ & 5 & 264 & $29,307.66$ & 5.69 & 795 & 1 \\
\hline ZjbZIP24 & XM_016037896.2 & $\mathrm{F}$ & 9 & 272 & $29,467.89$ & 6.21 & 819 & 2 \\
\hline ZjbZIP25 & XM_016010135.2 & G & UN & 409 & $43,539.79$ & 5.97 & 1230 & 12 \\
\hline ZjbZIP26 & XM_016033348.2 & G & 8 & 427 & $45,943.89$ & 6.54 & 1284 & 12 \\
\hline ZjbZIP27 & XM_016034676.2 & G & 8 & 359 & $37,885.71$ & 5.78 & 1080 & 11 \\
\hline ZjbZIP28 & XM_016044679.2 & $\mathrm{H}$ & 12 & 200 & $22,598.46$ & 9.69 & 603 & 4 \\
\hline ZjbZIP29 & XM_016030371.2 & $\mathrm{H}$ & 6 & 167 & $18,168.02$ & 9.64 & 504 & 4 \\
\hline ZjbZIP30 & XM_025069252.1 & $\mathrm{H}$ & UN & 177 & $19,743.88$ & 9.24 & 534 & 4 \\
\hline ZjbZIP31 & XM_025077206.1 & I & 9 & 589 & $64,322.77$ & 6.35 & 1770 & 4 \\
\hline ZjbZIP32 & XM_016022085.2 & I & 3 & 386 & $42,154.4$ & 5.85 & 1161 & 4 \\
\hline ZjbZIP33 & XM_016019180.2 & । & 2 & 470 & $49,855.63$ & 6.24 & 1413 & 4 \\
\hline ZjbZIP34 & XM_016012104.2 & I & UN & 427 & $46,237.98$ & 6.09 & 1284 & 4 \\
\hline ZjbZIP35 & XM_016010308.1 & I & UN & 339 & $36,967.19$ & 9.45 & 1020 & 4 \\
\hline ZjbZIP36 & XM_016036218.1 & S & 9 & 146 & $16,518.78$ & 8.11 & 441 & 1 \\
\hline ZjbZIP37 & XM_016016004.2 & S & UN & 229 & $25,550.97$ & 9.06 & 690 & 1 \\
\hline ZjbZIP38 & XM_016013151.2 & S & UN & 146 & $16,504.75$ & 8.11 & 441 & 1 \\
\hline ZjbZIP39 & XM_016024994.2 & S & 4 & 196 & $22,785.29$ & 6.13 & 591 & 1 \\
\hline ZjbZIP40 & XM_016024530.2 & L & 4 & 307 & $34,443.98$ & 4.65 & 924 & 3 \\
\hline ZjbZIP41 & XM_016013429.2 & C & UN & 349 & $38,379.34$ & 5.49 & 1050 & 6 \\
\hline ZjbZIP42 & XM_016023464.2 & K & 4 & 373 & $41,886.53$ & 8.33 & 1122 & 4 \\
\hline ZjbZIP43 & XM_016011524.2 & M & UN & 364 & $40,092.99$ & 6.05 & 1095 & 11 \\
\hline ZjbZIP44 & XM_016027660.1 & M & 5 & 127 & $14,671.01$ & 9.96 & 384 & 1 \\
\hline ZjbZIP45 & XM_016046481.2 & J & UN & 518 & $57,828.84$ & 8.98 & 1557 & 1 \\
\hline
\end{tabular}


Table 2 The RSCU of 64 codons in ZjbZIPs

\begin{tabular}{|c|c|c|c|c|c|}
\hline \multirow{2}{*}{$\begin{array}{l}\text { First } \\
\text { codon }\end{array}$} & \multicolumn{4}{|c|}{ Second codon } & \multirow{2}{*}{$\begin{array}{l}\text { Third } \\
\text { codon }\end{array}$} \\
\hline & $\mathrm{T}$ & C & $A$ & G & \\
\hline \multirow[t]{4}{*}{$\mathrm{T}$} & $\mathrm{TTT}(1.58)$ & $\mathrm{TCT}(1.59)$ & TAT(1.43) & TGT(1.19) & $\mathrm{T}$ \\
\hline & $\pi \mathrm{TC}(0.42)$ & TCC(0.92) & $\operatorname{TAC}(0.57)$ & $\mathrm{TGC}(0.81)$ & C \\
\hline & TТ(0.73) & TCA(1.35) & $\operatorname{TAA}(0.60)$ & TGA(1.40) & A \\
\hline & TTG(1.65) & TCG(0.62) & $\operatorname{TAG}(1.00)$ & TGG(1.00) & G \\
\hline \multirow[t]{4}{*}{ C } & CTT(1.46) & CCT(1.47) & CAT(1.25) & CGT(0.69) & $\mathrm{T}$ \\
\hline & CTC(0.76) & $\operatorname{CCC}(0.55)$ & CAC(0.75) & CGC(0.49) & C \\
\hline & $\mathrm{CTA}(0.55)$ & CCA(1.36) & $\mathrm{CAA}(0.97)$ & CGA(0.68) & A \\
\hline & $\mathrm{CTG}(0.85)$ & $\operatorname{CCG}(0.61)$ & CAG(1.03) & CGG(0.56) & G \\
\hline \multirow[t]{4}{*}{ A } & ATT(1.37) & ACT(1.38) & $\operatorname{AAT}(1.23)$ & AGT(0.86) & $\mathrm{T}$ \\
\hline & ATC(0.88) & ACC(0.98) & $\mathrm{AAC}(0.77)$ & AGC(0.66) & C \\
\hline & $\operatorname{ATA}(0.75)$ & $\mathrm{ACA}(1.19)$ & $\operatorname{AAA}(0.90)$ & $\operatorname{AGA}(1.79)$ & A \\
\hline & $\operatorname{ATG}(1.00)$ & $A C G(0.45)$ & $\mathrm{AAG}(1.10)$ & AGG(1.80) & G \\
\hline \multirow[t]{4}{*}{ G } & GTT(1.73) & GCT(1.69) & GAT(1.34) & GGT(1.35) & $\mathrm{T}$ \\
\hline & GTC(0.61) & GCC(0.72) & GAC(0.66) & GGC(0.59) & C \\
\hline & GTA(0.66) & $\mathrm{GCA}(1.22)$ & GAA(0.97) & GGA(1.34) & A \\
\hline & GTG(1.00) & GCG(0.36) & GAG(1.03) & GGG(0.72) & G \\
\hline
\end{tabular}

further supported by the phylogenetic tree of bZIPs between jujube and apple (Additional file 1). In jujube, the A subfamily is the largest subfamily, while in the Arabidopsis, the $\mathrm{S}$ subfamily is the largest subfamily [34]. As expected, bZIP proteins from the same group tended to cluster together and were named following the same scheme.

Using MEME software, a total of 10 conserved motifs among ZjbZIPs was identified (Fig. 2), of which motif 1 and motif 5 were identified as the core conserved domains and constituted the leucine zipper region of bZIP (Additional file 2). The proteins in each subfamily contain the same conserved motifs, which further support the above result of phylogenetic tree. However, they also have different conserved motifs among various subfamilies. For example, in addition to the core conserved domains, the A subfamily also contains three conserved motifs (Motif 6, 8 and 9), which may be related to their different biological functions.

\section{The phylogenetic tree and line charts for a lineage of} gene groups for ZjbZIP genes

In order to further study the evolution pattern and direction of ZjbZIP genes, ZjbZIP26 and 29 were selected

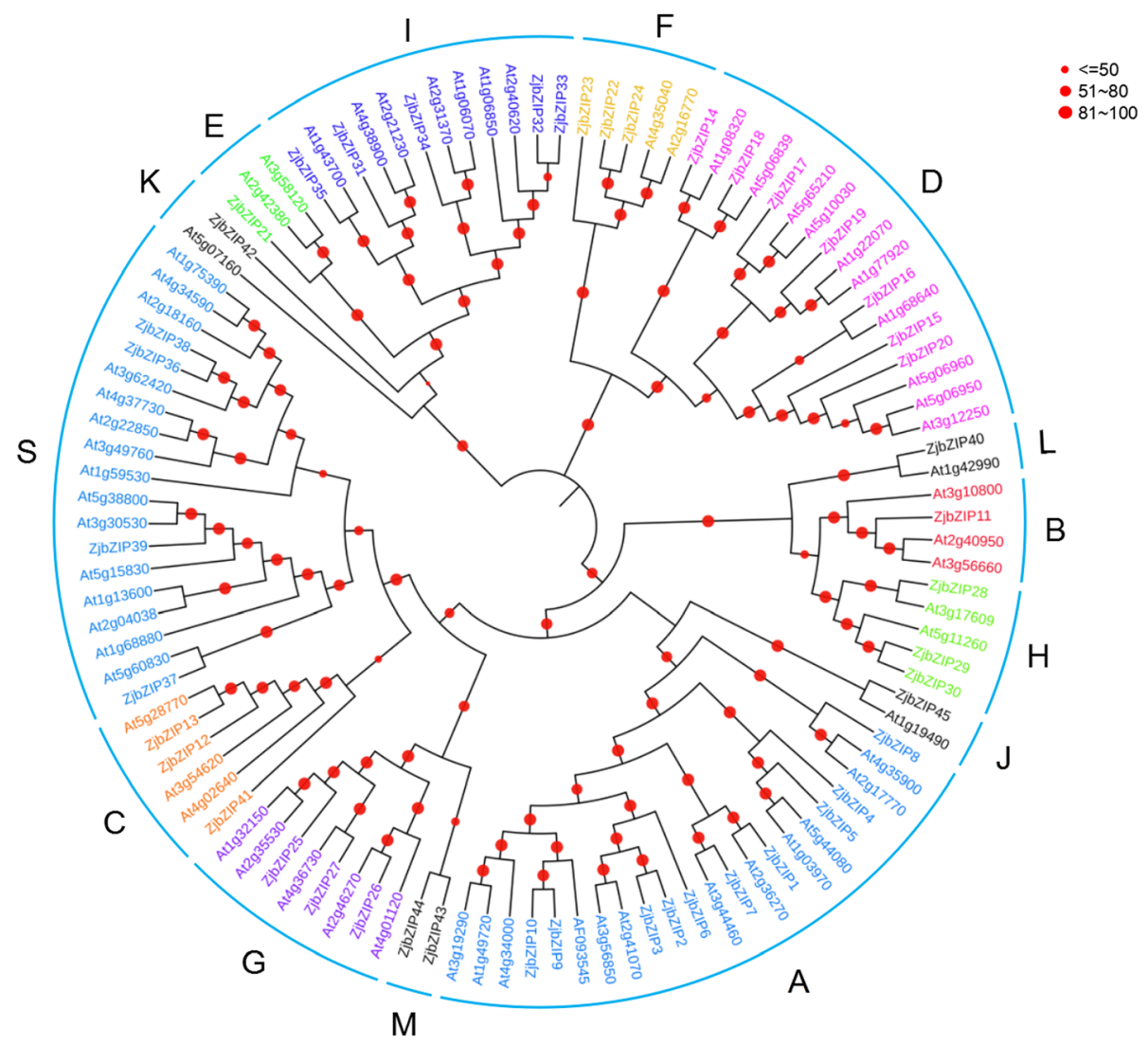

Fig. 1 The phylogenetic analysis of ZjbZIPs. The NJ tree was constructed from the protein sequences of ZjbZIPs using MEGA7 with 1000 bootstrap copies 


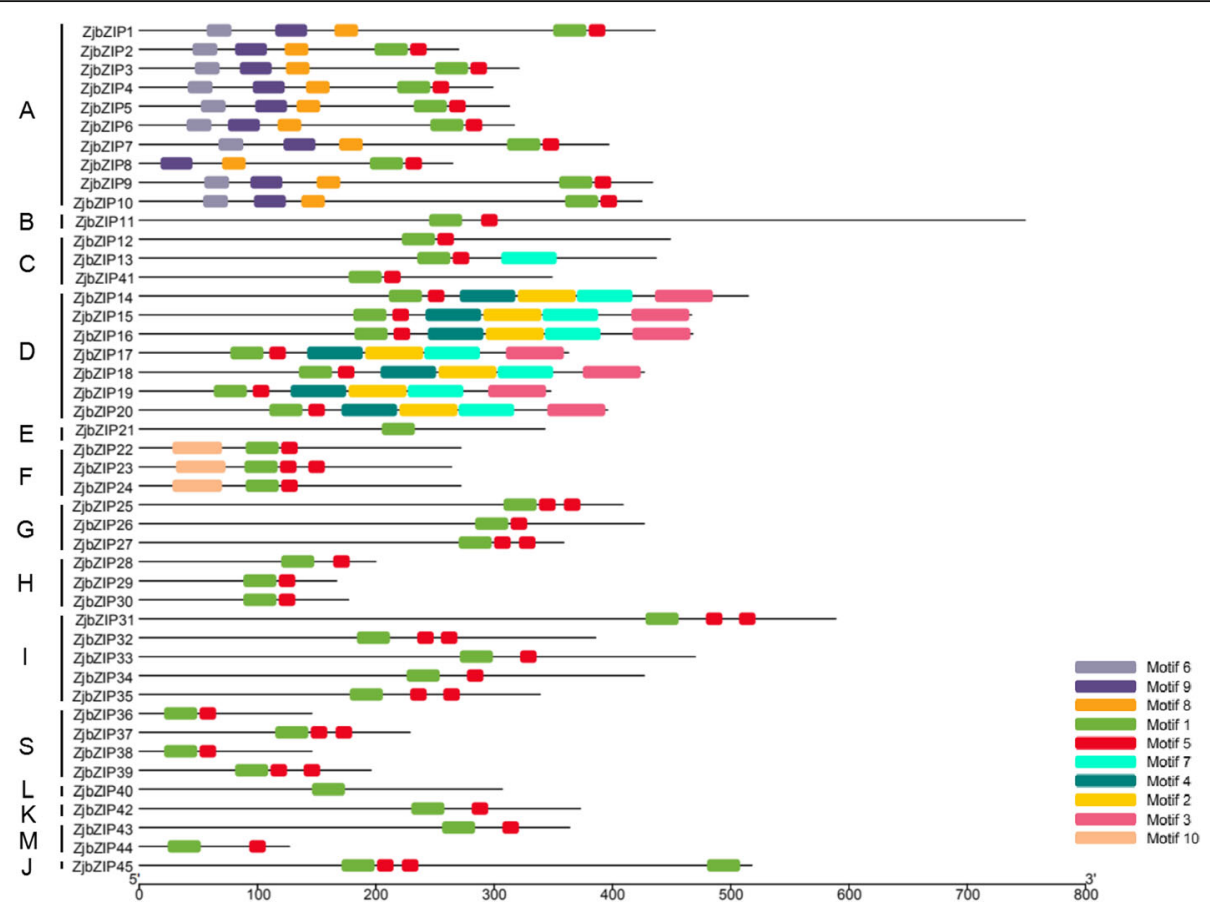

Fig. 2 Conserved motifs of ZjbZIP proteins. The motif in the ZjbZIP proteins was identified by using Multiple Em for Motif Elicitation (MEME). Ten conserved motifs were identified and displayed in different colors

to perform evolutionary analysis. ZjbZIP26 and ZjbZIP29 are homologous genes of GBF3 and HY5, respectively. And GBF3 and $H Y 5$ were proved to participate in various biological processes $[35,36]$.

As shown in the Fig. 3, two phylogenetic trees of ZjbZIP26 and -29 with 20 other genes showing high homology indices (HIs) in various species were constructed, respectively. The values of $H I s$ between all pairs in the two trees were all above 0.7, suggesting that they have similar amino acid sequences and might have conserved functions. To the phylogenetic tree of ZjbZIP29, three paralogous events were presumably occurred in a group of two genes (Cucumis sativus XP_004138731 and Cucumis melo NP_001284656), a group of two genes (Citrus clementina XP_006450470 and Citrus sinensis XP_006483336) and a group of two genes (Ziziphus jujuba XP_015885857 and XP_015868446) in Ziziphus jujuba. To the phylogenetic tree of ZjbZIP26, there were also five paralogous events.

Generally, along with evolutionary time the decrease of both the numbers of genes and species represents an orthologous event, and the number of genes decreases and the number of species is retained, which means a paralogous event [37]. For ZjbZIP26, along with evolutionary time, the numbers of genes (red line) and species (blue line) were both decreased in the timing between 0.619 and 0.632 of HIs, suggesting that an orthologous event happened; and only the numbers of genes (red line) were decreased in the timing between 0.570 and
0.582 of HIs, indicating that a paralogous event occurred. The two kinds of homologous events also found in ZjbZIP29 analysis. Therefore, the paralogous and orthologous duplication events should occurred during the evolutionary process of bZIPs.

The chromosomal location and gene structure of ZjbZIPs Of the 45 ZjbZIP genes, 34 were mapped to 10 pseudochromosomes in the jujube genome (Fig. 4), and 11 genes were unanchored. ZjbZIPs were not evenly distributed across the 12 chromosomes: there were 6 ZjbZIPs on both Chr. 9 and 12, and no genes were located on Chr. 1 or 11. Further analysis found that ZjbZIP1 and ZjbZIP2, ZjbZIP6 and ZjbZIP19, ZjbZIP7 and ZjbZIP14 are tandem repeat genes, indicating that some ZjbZIPs underwent gene duplication during jujube evolution to increase the number of genes and enhance their biological functions.

Additionally, the gene structure within the same subfamily was highly conserved (Additional file 3 ). We found that the genes in the C, D and G subfamilies contained more introns than did the genes in the other groups.

\section{Expression patterns of ZjbZIPs in various organs}

To investigate the organ-specific expression of the ZjbZIP genes, the expression of 30 ZjbZIPs were analyzed in five organs of jujube and wild jujube by RT-PCR (Fig. 5, Additional file 4). It was shown that most 

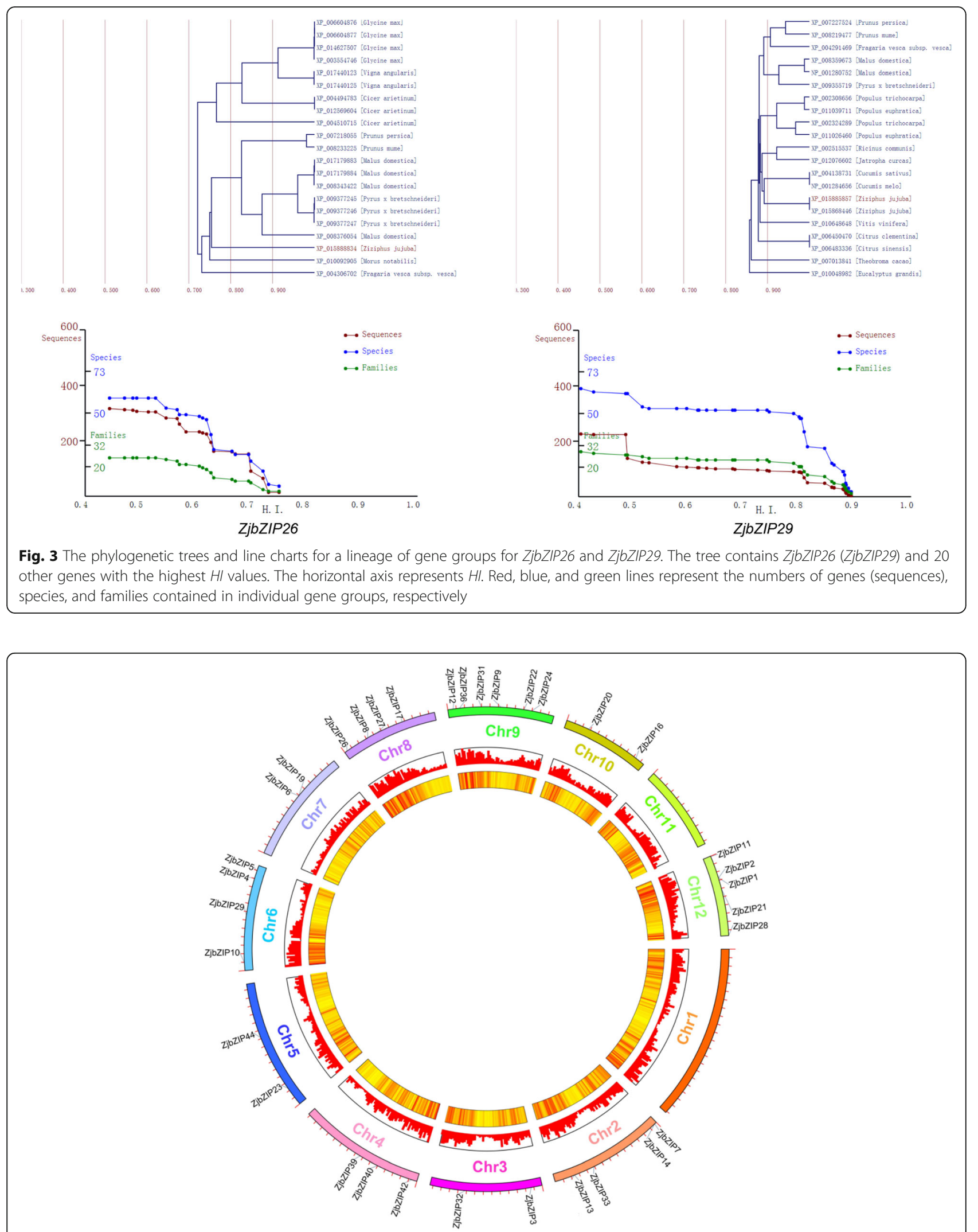

Fig. 4 The chromosomal location of 34 ZjbZIPs. Genes are mapped to jujube chromosomes by the TBtools. The chromosomes of jujube are arranged in a circle 


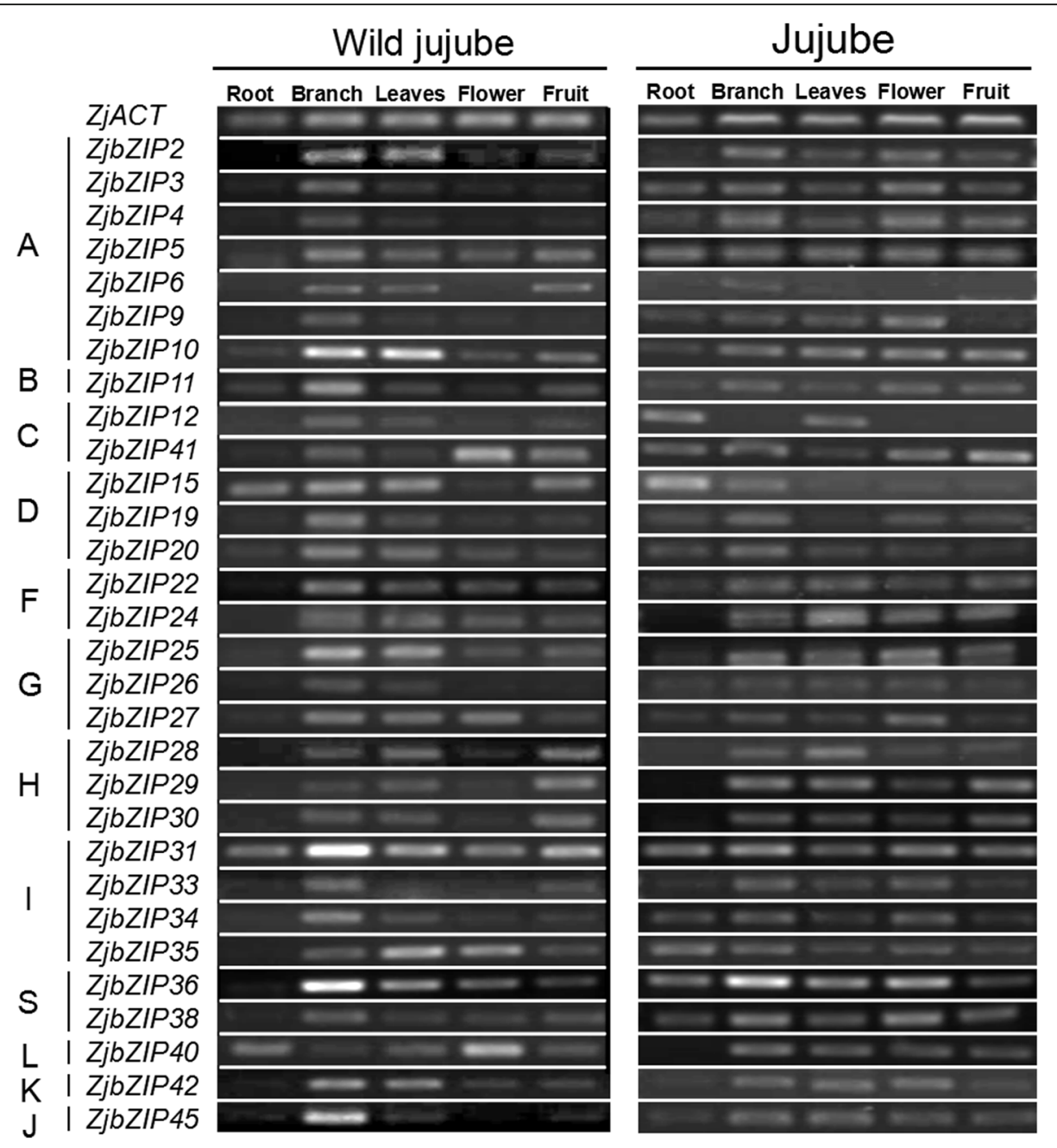

Fig. 5 Expression patterns of 30 ZjbZIPs in five tissues of wild jujube and jujube by RT-PCR. ZjACT was used as an internal control. Left: wild jujube, from left to right: root, branch, leave, flower, and fruit. Right: jujube, from left to right: root, branch, leave, flower, fruit

ZjbZIPs were expressed in at least four organs, indicating that ZjbZIPs should involve in the development process of various organs in jujube and wild jujube. The expression of most ZjbZIP genes in the same subfamily showed similar patterns, suggesting that these genes in the same subfamily might have conserved functions.

In wild jujube, some ZjbZIP genes display a special expression pattern, in which ZjbZIP10, - 25, - 31, - 36 were highly expressed in branch and leaf, indicating that these genes should play some roles in the development of the two organs. Compared with jujube, the expression levels of some genes, such as ZjbZIP3, - 5, - 12, - 34, $35,-36,-38$, and -41 , were significantly decreased in root of wild jujube, and only three genes, ZjbZIP15, 31, - 40, showed lower expression (Fig. 5). These ZjbZIP genes may be related to their differing functions in root between wild jujube and jujube. We also found most of ZjbZIPs were expressed with varying degrees in fruit, and these genes should be candidate genes for further investigating in jujube fruit development. The broad and divergent expression patterns indicated that the ZjbZIPs should have multiple functions in jujube growth and development.

\section{ZjbZIPs involvement in jujube fruit development}

Based on organ-specific expression (Fig. 5), the highly expressed genes in fruit were further investigated at five development stages of jujube fruit. It is noteworthy that almost all of the ZjbZIPs tested were highly expressed at the first two stages and showed similar trends in both of the two cultivars (Fig. 6), indicating that these genes were positively involved in the fruit enlargement process. In other word, ZjbZIPs should play some significant roles in jujube fruit development.

Especially, ZjbZIP28, - 29, - 30, - 36, - 38 showed the obvious increase in expression at the early white mature fruit stage (EWM), which period is just the fruit rapid expanding stage. These genes were identified as 


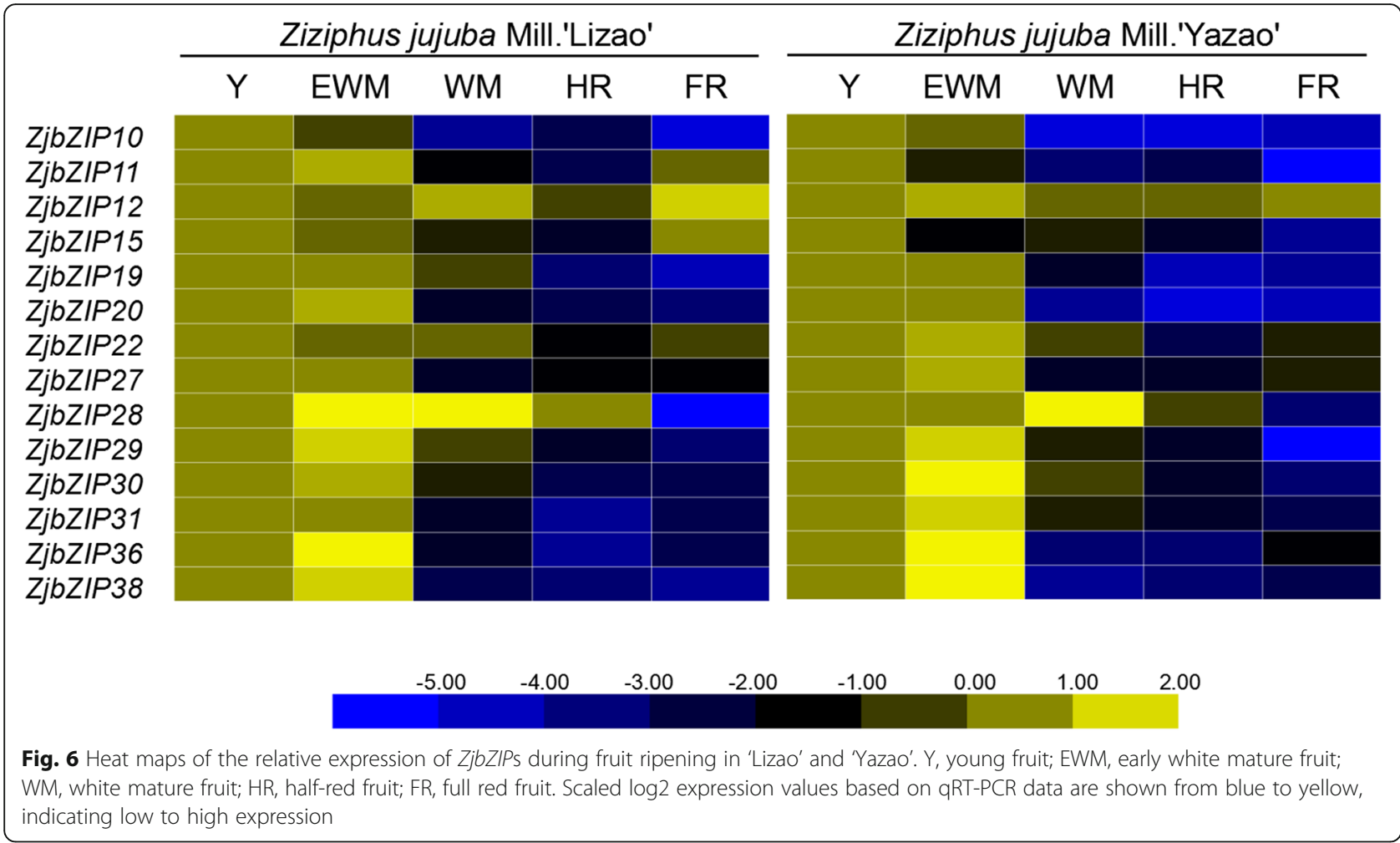

candidate genes for further study, in terms of their functions in fruit development.

\section{ZjbZIP protein-protein interaction network prediction}

Based on their orthologs in Arabidopsis, ZjbZIPs were predicted to interact with each other by STRING (Fig. 7a), which was in accordance with previous reports that the binding activity of bZIPs depends upon the formation of homodimers or heterodimers among bZIPs [38]. Several interactions including ZjbZIP27 and ZjbZIP28, ZjbZIP29 and ZjbZIP28, were further confirmed by yeast two-hybrid in Fig. 7b (Additional file 4). As shown in Fig. 7a, both TGA9 (homolog of ZjbZIP14) and TGA10 (homolog of ZjbZIP18) are involved in the regulation of anther development [39], B202H1 (homo$\log$ of ZjbZIP12) can interact with BZIP53 (homolog of ZjbZIP36, ZjbZIP38 and ZjbZIP44) to promote their expression in seeds and are involved in defense responses [40], and the interaction between GBF4 (homolog of ZjbZIP4) and bZIP68 (homolog of ZjbZIP25) is regulated by light or other hormones [41].

\section{The expression of bZIP genes under abiotic and biotic stress}

According to protein-protein interaction predictions and previous studies, 11 ZjbZIPs were selected for further validation under abiotic stress, including lowtemperature and salt stresses (Additional file 5). Under salt stress, the expression levels of ZjbZIP10, 11, 23 and 40 (Additional file 5) increased with prolonged treatment time. The expression of ZjbZIP10 increased significantly after $6 \mathrm{~h}$ of treatment and peaked at $48 \mathrm{~h}$ (Fig. 8a), and ZjbZIP4O expression peaked at $1 \mathrm{~h}$ of treatment but then decreased. The expression of ZjbZIP3 showed no difference under salt stress. We also found that the expression of ZjbZIP29, ZjbZIP36 and 38 increased markedly under low-temperature stress (Additional file 5 and Fig. 8b), and the expression of other three genes (ZjbZIP17, 22 and 24) also increased in varying degrees (Additional file 5).

Jujube withes' broom (JWB), which is caused by phytoplasma, is a destructive disease that affects jujube production. Since bZIP genes have multiple functions in plants, whether they participate in jujube-phytoplasma interactions remains unclear. Among the 17 ZjbZIPs identified (Fig. 9), the expression of ZjbZIP3 and 12 in diseased leaves was significantly lower than that in healthy leaves. Moreover, the ZjbZIP11, 15, 17, 18, 19, 20 and 26 genes were highly expressed in diseased leaves. These results suggested that these ZjbZIPs participate in jujube-phytoplasma interactions and play different roles.

\section{Discussion}

In this study, a total of 45 bZIP genes were identified in the jujube genome. A previous genome evolution study 


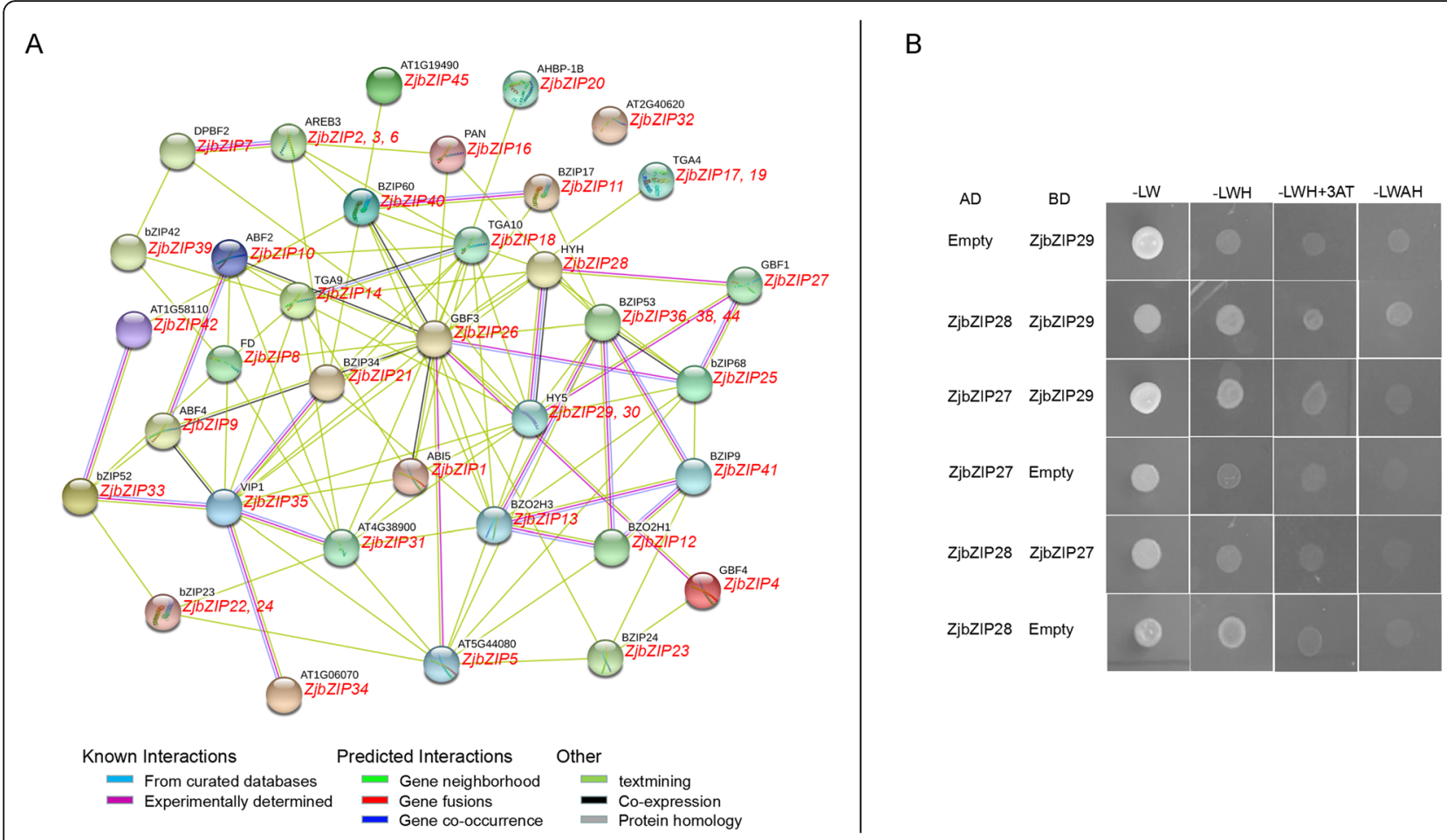

Fig. 7 a: Protein-protein interaction network for ZjbZIPs based on their orthologs in Arabidopsis. This network was predicted by online software STRING. The ZjbZIP proteins were shown in the red font below with the Arabidopsis orthologs. b: Yeast two-hybrid screening of ZjbZIP27, ZjbZIP28 and ZjbZIP29

showed that Chinese jujube is closely related to species of the Rosaceae family [24], so the numbers of bZIPs from two Rosaceae species (apple and peach) [42], grape and Arabidopsis were compared with the number of ZjbZIPs. Compared with the number in some other plant species, the smaller number of bZIP genes in jujube and peach should be caused by the occurrence of only one whole genome duplication (WGD) event during the species evolution. Meanwhile, the divergence of gene number in same gene family of various species also relate to their evolutionary differences or the genome size [28].

In Arabidopsis, various subfamilies of AtbZIPs contain different conserved domains and perform various biological functions. For example, most members of the class A subfamily are involved in ABA pathway and abiotic stress responses, the class D members mainly play some roles in pathogen resistance and plant development, and the class S subfamily is involved in carbohydrate metabolism [34]. Here, the ZjbZIPs are highly homologous to sequences of the corresponding subfamilies in Arabidopsis, indicating that ZjbZIPs also might play different functions as a result of different conserved domains.

There are 10 TGA family members in Arabidopsis (Additional file 6), which are divided into five categories.
A number of studies have shown that members of the TGA family play an important role in defense against biological and necrotizing pathogens $[43,44]$. One member of the TGA family was found to interact with an ankyrin-repeat protein, a nonexpressor of the pathogenassociated (PR) gene (NPR1), which is a key component of the SA defense signaling pathway; SA is a key signaling molecule involved in plant resistance $[45,46]$. Moreover, TGA members participate in mitotic reactions, regulate the growth and development of organisms, and play an important role in flower development [43]. Homology analysis and phylogenetic tree analysis indicated that subfamily $\mathrm{D}$ of ZjbZIP family is highly homologous to the TGA family in Arabidopsis (Additional file 6). It showed that ZjbZIP14 and TGA9 (At1g08320), ZjbZIP18 and TGA10 (At5g06839), ZjbZIP19 and TGA4 (At5g10030) were homologous (Additional file 6), suggesting that they might have similar biological functions and participate in pathogen defense reactions. Moreover, ZjbZIP17, - 18, - 19 and - 20, which are highly homologous to the TGA members in Arabidopsis, showed differential expression at the early stage of phytoplasma infection (Fig. 9). Phytoplasma causes one of serious disease in jujube production, named Jujube Witches' Broom. Thus, these genes should involve in jujubephytoplasma interaction. 

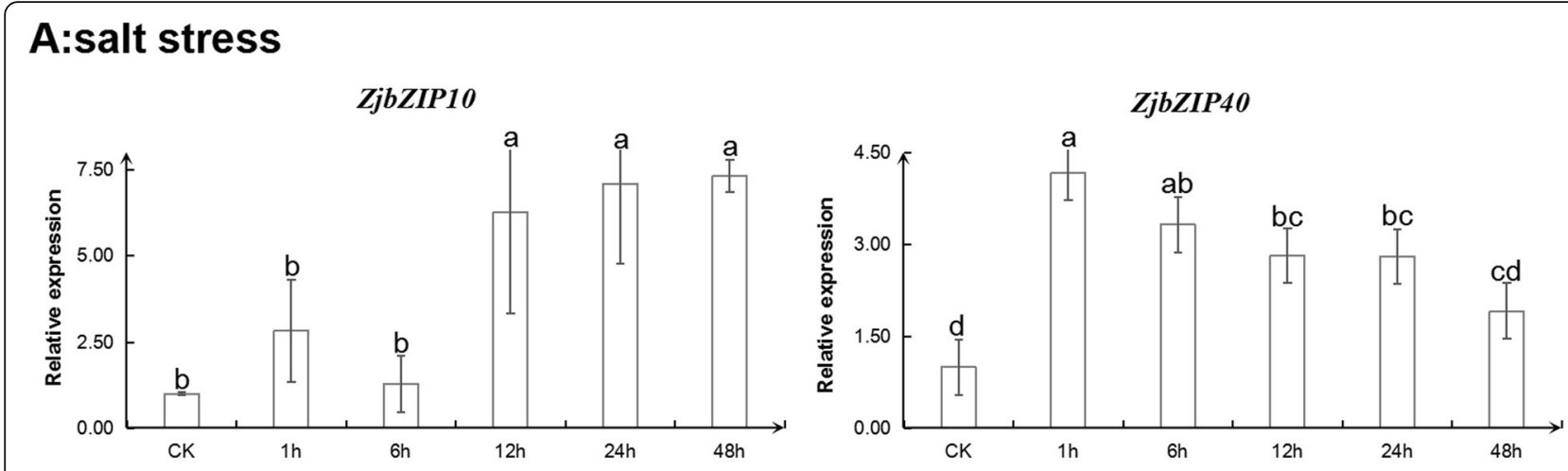

\section{B:Iow temperature stress}
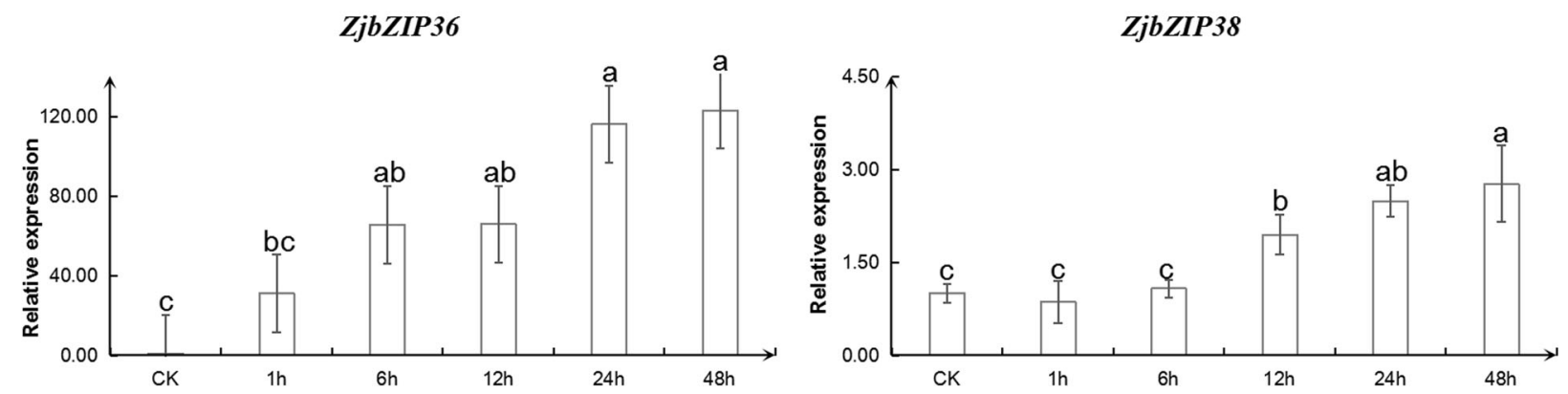

Fig. 8 The relative expression of ZjbZIPs under abiotic stress. a: The relative expression of two ZjbZIPs under salt stress. $\mathbf{b}$ : The relative expression of two ZjbZIPs under low temperature stress. All statistical analyses were performed with SPSS software 17.0. Duncan's multiple range tests were used to assess differences between treatments. Different letters mean significant difference at 0.05 levels between the corresponding treatments

AtbZIPs can recognize and bind to ABREs within promoters, which are named ABRE- binding factors (ABFs)/ ABRE-binding proteins (AREBs) [9]. They play a key role in the regulation of the expression of downstream stress-responsive genes involved in ABA signaling. Genetic transformation of $\mathrm{ABF} / \mathrm{ABRE}$ transcription factors has been suggested to be an effective approach for engineering stress-tolerant plants [47]. In this study, we also found that the expression level of ZjbZIP10 was increased with time under salt stress. Thus, ZjbZIP10 may have the function in terms of involvement in the resistance to salt stress. In addition, the expression of ZjbZIP36 was significantly induced under lowtemperature stress, indicating that it might be involved in the response to cold stress.

Overall, all the results imply that ZjbZIPs play multiple roles in jujube development and under biotic and abiotic stresses. Further function verifications are worthwhile and need to reveal their regulation metabolism in detail.

\section{Conclusions}

The bioinformatics analyses of 45 ZjbZIPs were firstly investigated in this study. Meanwhile, their expression patterns in jujube and wild jujube were measured by qPCR during fruit ripening and in the response to phytoplasma and abiotic stresses, and some candidate genes involved in these processes were screened out. The protein interactions among ZjbZIPs were predicted, which provide useful information for further functional studies to elucidate their regulation mechanism.

\section{Methods}

\section{Plant materials}

The Chinese jujube and wild jujube trees used in this study were cultivated at the Experimental Station of Chinese Jujube, Hebei Agricultural University, Baoding, China. They are very common fruit trees in China and are not endangered species. No specific permits were required for the sample collection.

Five organs (roots, branches, leaves, flowers and fruits) collected from three jujube trees and three wild jujube trees were used for organ-specific expression analysis. In general, jujube fruit development can be divided into five typical stages: the young fruit stage $(\mathrm{Y})$, early white mature fruit stage (EWM), white mature fruit stage (WM), half-red fruit stage (HR) and full-red fruit stage (FR). The first two stages of jujube fruit development (young fruit stage (Y) and early white mature fruit stage $(E W M))$ are crucial to fruit enlargement, and the later three stages are important to fruit maturity. Z. jujuba 


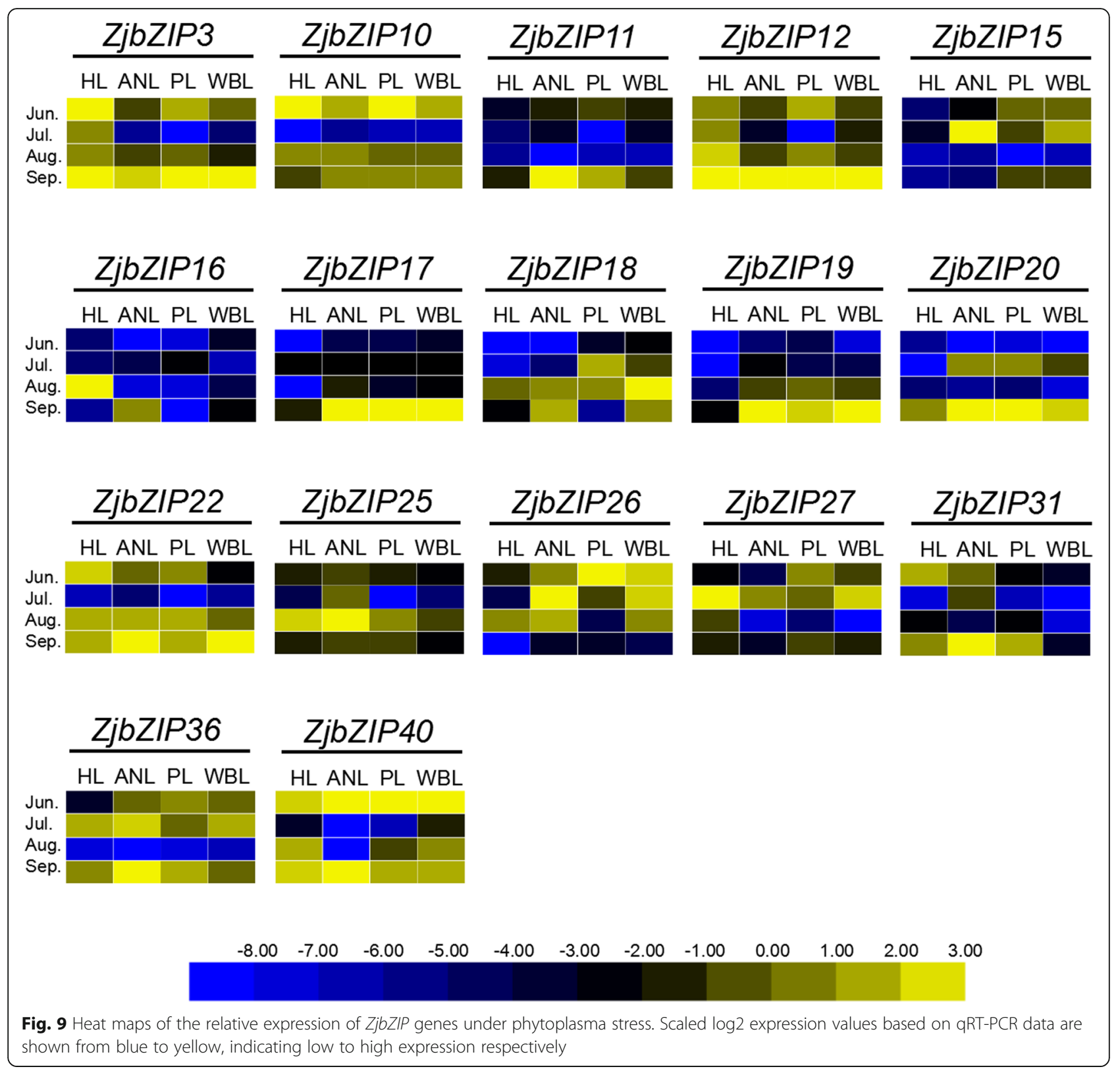

Mill. 'Lizao' and 'Yazao' are two jujube fresh cultivars, which fruit display five typical developmental stages and suitable to study the fruit development. Thus, fruit samples of the two cultivars were taken at the five developmental stages and used to investigate the expression patterns of ZjbZIPs. Each treatment consisted of three biological replicates.

Three healthy trees and three JWB diseased trees of $Z$. jujuba Mill. 'Dongzao' were used to collect the leave samples at four stages (June, July, August and September). There are four kinds of leaves, i.e., healthy leaves (HL), apparently normal leaves (ANL), phyllody leaves (PL), and witches' broom leaves (WBL). All treatments consisted of three biological replicates. All fresh samples were frozen in liquid nitrogen immediately, and then stored at $-80^{\circ} \mathrm{C}$ for RNA extraction.

Cold- and salt-stress treatments were performed on the callus tissues of $Z$. jujuba Mill. 'Guanyangchangzao'. For the cold treatment, calli at same growth stage and condition were transferred at $4{ }^{\circ} \mathrm{C}$ and then collected within $0,1,6,12,24$, and $48 \mathrm{~h}$. Calli incubated at $25^{\circ} \mathrm{C}$ were collected and used as negative controls [26]. For the salinity treatment, callus tissues were subjected to a $150 \mathrm{mM} \mathrm{NaCl}$ and $\mathrm{NaHCO}_{3}$ $\mathrm{NaOH}$ solution ( $\mathrm{pH} 9.5$ ) for $0,1,6,12,24$, and $48 \mathrm{~h}$. Samples subjected to sterile water treatment rather than the $\mathrm{NaCl}$ and $\mathrm{NaHCO}_{3}-\mathrm{NaOH}$ solution were used as negative controls [48]. 
Identification and protein structure analysis of ZjbZIPs A hidden Markov model (HMM) file of the bZIP domain (PF00170) was downloaded (Pfam 31.0, https:// pfam.xfam.org/) and used as the template to identify ZjbZIP sequences in the jujube genome [24]. Above sequences were further confirmed by using the Plant Transcription Factor Database (PlantTFDB, http:// planttfdb.cbi.pku.edu.cn/) and the online CD search tool in NCBI database (https://www.ncbi.nlm.nih.gov/). Some characters of the ZjbZIP genes were also predicted by the bioinformatical tools [49]. GC content and RSCU were performed by software codonW1.4.4 (http:// codonw.sourceforge.net/). The conserved motifs of ZjbZIP proteins were detected by MEME (http://memesuite.org/).

The chromosomal location and gene structure of ZjbZIPs The chromosomal locations of the ZjbZIP genes and their tandem duplications were analyzed as previously described [49, 50]. TBtools (http://cj-chen.github.io/ tbtools/) was used to draw a circle graph. The gene structure of ZjbZIPs was predicted by website GSDS (http://gsds.cbi.pku.edu.cn/) [49, 51].

\section{Multiple sequence alignment and phylogenetic tree construction}

The multiple sequence alignment results were analyzed by using DNAMAN 8.0 software. A phylogenetic tree consisting of 45 ZjbZIPs was constructed based on their conserved domains. bZIP proteins from Arabidopsis thaliana and Malus were downloaded from the NCBI (https://www.ncbi.nlm.nih.gov/) and PlantTFDB (http:// planttfdb.cbi.pku.edu.cn/) (Additional file 7) and used to construct the phylogenetic tree $[52,53]$. In addition, the phylogenetic trees and line charts for a lineage of gene groups for ZjbZIP26 and 29 were analyzed in the Gcorn plant database (http://www.plant.osakafu-u.ac.jp/ kagiana/gcorn/p/) [37].

\section{RNA isolation and expression analysis}

The procedures of total RNA isolation, detection and cDNA synthesis were performed according to previous methods [49]. Both semiquantitative PCR and qRT-PCR were used to measure the expression of ZjbZIPs. The primer sequences were listed in Additional file 8. The PCR reaction system and procedures in the study were implemented as the method described [49, 54, 55]. Three biological replicates were analyzed for each treatment.

All statistical analyses were performed with SPSS software 17.0. Duncan's multiple range tests were used to assess differences among different treatment times. Different letters mean significant difference at 0.05 levels between the corresponding treatments.

\section{Protein-protein interaction predictions}

45 ZjbZIP protein sequences were used as the targets and their orthologs of Arabidopsis thaliana were appointed as references, and the STRING website (https://string-db.org/) was employed to predict proteinprotein interactions [49]. Yeast two-hybrid screening $(\mathrm{Y} 2 \mathrm{H})$ [56] was further applied to verify several interactions.

\section{Supplementary information}

Supplementary information accompanies this paper at https://doi.org/10 1186/s12864-020-06890-7.

Additional file 1: Fig. S1. The phylogenetic analysis of bZIP proteins of Ziziphus jujuba Mill and Malus domestica. The NJ tree was constructed from the bZIP protein sequences using MEGA7 with 1000 bootstrap copies.

Additional file 2: Fig. S2. The conserved motifs of ZjbZIP proteins.

Additional file 3: Fig. S3. The gene structure of 45 ZjbZIPs in Chinese jujube. Introns and exons are represented by black lines and red boxes respectively and upstream/downstream are represented by green boxes.

Additional file 4. A: All original and full-length gels in Fig. 5; B: All original and blot images in Fig. 7b.

Additional file 5: Fig. S4. The relative expression of ZjbZIPs under salt stress and low temperature stress.

Additional file 6: Fig. S5. A: The phylogenetic analysis of bZIP proteins of Ziziphus jujuba and Arabidopsis thaliana. The NJ tree was constructed from the protein sequences of ZjbZIPs and AtbZIPs using MEGA7 with 1000 bootstrap copies. B: The protein-protein interaction analysis of two ZjbZIPs by STRING database.

Additional file 7: Table S1. The bZIP transcription factors in Arabidopsis and apple used in this study. (XLS $38 \mathrm{~kb}$ )

Additional file 8: Table S2. The primers of ZjbZIPs used in this study. (XLS 29 kb)

\section{Abbreviations}

TFs: transcription factors; bZIP: basic helix-loop-helix; TGA: TGACG motifbinding factor; HIs: high homology indices; qRT-PCR: quantitative real-time PCR; HMM: hidden Markov model; JWB: jujube witches' broom disease; ANL: apparently normal leaves; PL: phyllody leaves; WBL: witches' broom leaves; HL: healthy leaves; Y: young fruit stage; EWM: early white mature fruit stage; WM: white mature fruit stage; HR: half-red fruit stage; FR: full-red fruit stage

\section{Acknowledgements}

Not applicable.

\section{Authors' contributions}

JZ designed the research; YZ, WG, HL and JZ performed the experiments, analyzed the data and wrote the paper. $Y Z, H L, Z L$ and $M L$ participated in the data analysis. YZ, YW and DL collected the samples. YZ, CX and WG performed RT-PCR and GRT-PCR experiments. All authors read and approved the final the manuscript.

\section{Funding}

Supported by the National Key R\&D Program Project Funding (2018YFD1000607), the National Natural Science Foundation of China (31772285), Foundation for 100 Innovative Talents of Hebei Province (SLRC2019031), Hebei Distinguished Young Scholar (C201620 4145), and Hebei Special Funds for Intellectual Introduction and Talent Training (A20160 02054). These funding bodies had no role in the design of the study, sample collection, analysis or interpretation of data and in writing the manuscript. 


\section{Availability of data and materials}

All data and materials used in this study are publicly available. All accession numbers deposited in the NCBI and PlantTFDB have been listed in Table 1 and additional file 7. The other accession numbers (XP_004138731, NP_001284656, XP_006450470, XP_006483336, At1 g08320, At5g06839 and At5g10030) and related web links are included in the manuscript.

\section{Ethics approval and consent to participate}

Not applicable.

\section{Consent for publication}

Not applicable.

\section{Competing interests}

The authors declare that they have no competing interests.

\section{Author details}

${ }^{1}$ College of Life Science, Hebei Agricultural University, Baoding, China. ${ }^{2}$ Hebei Key Laboratory of Plant Physiology and Molecular Pathology, Hebei Agricultural University, Baoding, China. ${ }^{3}$ Pomology Institute, Shanxi Academy of Agricultural Sciences, Taigu, China. ${ }^{4}$ Research Center of Chinese Jujube, Hebei Agricultural University, Baoding, China.

Received: 7 February 2020 Accepted: 7 July 2020

Published online: 14 July 2020

\section{References}

1. Kong S, Park SY, Lee YH. Systematic characterization of the bZIP transcription factor gene family in the rice blast fungus, Magnaporthe oryzae. Environmental Microbiol. 2015;17(4):1425-43.

2. Yin W, Cui $P$, Wei W, et al. Genome-wide identification and analysis of the basic leucine zipper (bZIP) transcription factor gene family in Ustilaginoidea virens. Genome. 2017;60(12):1051-9.

3. Jiao Z, Rongrong G, Chunlei G, et al. Evolutionary and expression analyses of the apple basic leucine zipper transcription factor family. Front Plant Sci. 2016;7(3):376

4. Llorca CM, Potschin M, Zentgraf U. bZIPs and WRKYs: two large transcription factor families executing two different functional strategies. Front Plant Sci. 2014;5(4):169.

5. Que F, Wang GL, Huang Y, et al. Genomic identification of group a bZIP transcription factors and their responses to abiotic stress in carrot. Genet Mol Res. 2015;14(4):13274-88.

6. Vanitha J, Ramachandran S. Genome-wide expansion and expression divergence of the basic Leucine zipper transcription factors in higher plants with an emphasis on sorghum. J Integr Plant Biol. 2011;53(3):212-31.

7. Noman A, Liu Z, Aqeel M, et al. Basic leucine zipper domain transcription factors: the vanguards in plant immunity. Biotechnol Lett. 2017;39(3):1779_ 91.

8. Woo LC, Woonhee B, Chul LS. Roles of pepper bZIP protein CaDILZ1 and its interacting partner RING-type E3 ligase CaDSR1 in modulation of drought tolerance. Plant J. 2018:96:452-67.

9. Zhao BY, Hu YF, Li J, et al. BnaABF2, a bZIP transcription factor from rapeseed (Brassica napus L.), enhances drought and salt tolerance in transgenic Arabidopsis. Botanical Studies. 2016;57(1):12.

10. Sirichandra C, Davanture M, Turk BE, et al. The Arabidopsis ABA-activated kinase OST1 phosphorylates the bZIP transcription factor ABF3 and creates a 14-3-3 binding site involved in its turnover. PLoS One. 2010;5(11):e13935.

11. Christoph W, Lorenzo P, Jebasingh S, et al. The Arabidopsis bZIP11 transcription factor links low-energy signalling to auxin-mediated control of primary root growth. PLoS Genet. 2017;13(2):e1006607.

12. Jain P, Shah K, Sharma N, et al. A-ZIP53, a dominant negative reveals the molecular mechanism of heterodimerization between bZIP53, bZIP10 and bZIP25 involved in Arabidopsis seed maturation. Sci Rep. 2017;7(1):14343.

13. Ling $Z$, Hao $Y$, Shunquan $L$, et al. Molecular characterization of FT and FD homologs from Eriobotrya deflexa Nakai forma koshunensis. Front Plant Sci. 2016;7:8.

14. Yoshida T, Fujita Y, Maruyama K, et al. Four Arabidopsis AREB/ABF transcription factors function predominantly in gene expression downstream of SnRK2 kinases in abscisic acid signaling in response to osmotic stress. Plant Cell Environ. 2015;38(1):35-49.
15. Meng XB, Zhao WS, Lin RM, et al. Identification of a novel rice bZIP-type transcription factor gene, OsbZIP1, involved in response to infection of Magnaporthe grisea. Plant Mol Biol Report. 2005;23(3):301-2.

16. Després C, Chubak C, Rochon A, et al. The Arabidopsis NPR1 disease resistance protein is a novel cofactor that confers redox regulation of DNA binding activity to the basic domain/leucine zipper transcription factor TGA1. Plant Cell. 2003;15(9):2181-91.

17. Lebel $E$, Heifetz $P$, Thorne $L$, et al. Functional analysis of regulatory sequences controlling PR-1 gene expression in Arabidopsis. Plant J. 1998; 16(2):223-33.

18. Zhang Y, Fan W, Kinkema M, et al. Interaction of NPR1 with basic leucine zipper protein transcription factors that bind sequences required for salicylic acid induction of the PR-1 gene. Proc Natl Acad Sci. 1999;96(11):6523-8.

19. Zhang $Y$, Tessaro MJ, Lassner M, et al. Knockout analysis of Arabidopsis transcription factors TGA2, TGA5, and TGA6 reveals their redundant and essential roles in systemic acquired resistance. Plant Cell. 2003;15(11):264753.

20. Zander M, La Camera S, Lamotte O, et al. Arabidopsis thaliana class-II TGA transcription factors are essential activators of jasmonic acid/ethyleneinduced defense responses. Plant J. 2010;61(2):200-10.

21. Johnson C, Boden E, Arias J. Salicylic acid and NPR1 induce the recruitment of trans-activating TGA factors to a defense gene promoter in Arabidopsis. Plant Cell. 2003;15(8):1846-58.

22. Wang D, Amornsiripanitch N, Dong X. A genomic approach to identify regulatory nodes in the transcriptional network of systemic acquired resistance in plants. PLoS Pathog. 2006;2(11):e123.

23. Kesarwani M, Yoo J, Dong X. Genetic interactions of TGA transcription factors in the regulation of pathogenesis-related genes and disease resistance in Arabidopsis. Plant Physiol. 2007;144(1):336-46.

24. Liu M, Zhao J, Cai Q, Liu G, Wang J, Zhao Z, et al. The complex jujube genome provides insights into fruit tree biology. Nat Commun. 2014;5:5315.

25. Hou L, Zhang Z, Dou S, et al. Genome-wide identification, characterization, and expression analysis of the expansin gene family in Chinese jujube (Ziziphus jujuba mill.). Planta. 2019;249(3):815-29.

26. Mingxin $G$, Shipeng $L$, Shan $T$, et al. Transcriptome analysis of genes involved in defense against alkaline stress in roots of wild jujube (Ziziphus acidojujuba). PLoS One. 2017;12(10):e0185732.

27. Wolfgang DL, Snoek BL, Berend S, et al. The Arabidopsis bZIP transcription factor family-an update. Curr Opin Plant Biol. 2018;45:36-49.

28. Liu J, Chen N, Chen F, et al. Genome-wide analysis and expression profile of the bZIP transcription factor gene family in grapevine (Vitis vinifera). BMC Genomics. 2014;15(1):281.

29. Yang Z, Sun J, Chen Y, et al. Genome-wide identification, structural and gene expression analysis of the bZIP transcription factor family in sweet potato wild relative Ipomoea trifida. BMC Genet. 2019;20(1):41.

30. Zhang L, Zhao J, Feng C, et al. Genome-wide identification, characterization of the MADS-box gene family in Chinese jujube and their involvement in flower development. Sci Rep. 2017;7(1):1025.

31. Lin $T$, Ni Z, Shen $M$, et al. High-frequency codon analysis and its application in codon analysis of tobacco. J Xiamen University Natural Sci. 2002;41(5): $551-4$.

32. Feng $C, X u$ C, Wang $Y$, et al. Codon usage patterns in Chinese bayberry (Myrica rubra) based on RNA- seq data. BMC Genomics. 2013;14(1):732.

33. Lim SK, Sigmund CD, Gross KW, et al. Nonsense codons in human betaglobin mRNA result in the production of mRNA degradation products. Mol Cell Biol. 1992;12(3):1149-61.

34. Jakoby M, Weisshaar B, Drögelaser W, et al. bZIP transcription factors in Arabidopsis. Trends Plant Sci. 2002;7(3):106-11.

35. Ramegowda V, Gill US, Sivalingam PN, et al. GBF3 transcription factor imparts drought tolerance in Arabidopsis thaliana. Sci Rep. 2017;7(1):1-13.

36. Lee J, He K, Stolc V, et al. Analysis of transcription factor HY5 genomic binding sites revealed its hierarchical role in light regulation of development. Plant Cell. 2007;19(3):731-49.

37. Ogata Y, Kimura N, Sano R. Gcorn plant: a database for retrieving functional and evolutionary traits of plant genes. Plant Physiol. 2019;180(2):732-42.

38. Rushing AW, Rushing B, Hoang K, Sanders SV, Pe'loponèse J-M Jr, Polakowski N, et al. HTLV-1 basic leucine zipper factor protects cells from oxidative stress by upregulating expression of Heme Oxygenase I. PLoS Pathogens. 2019;15(6):e1007922.

39. Murmu J, Bush MJ, Delong C, et al. Arabidopsis basic leucine-zipper transcription factors TGA9 and TGA10 interact with floral glutaredoxins 
ROXY1 and ROXY2 and are redundantly required for anther development. Plant Physiol. 2010;154(3):1492-504.

40. Alonso R, Onate-Sanchez L, Weltmeier F, et al. A pivotal role of the basic Leucine zipper transcription factor bZIP53 in the regulation of Arabidopsis seed maturation gene expression based on hetero- dimerization and protein complex formation. Plant Cell. 2009;21(6):1747-61.

41. Terzaghi WB, Bertekap RL, Cashmore AR. Intracellular localization of GBF proteins and blue light- induced import of GBF2 fusion proteins into the nucleus of cultured Arabidopsis and soybean cells. Plant J. 1997;11(5):96782.

42. Sun MY, Fu XL, Tan QP, et al. Analysis of basic leucine zipper genes and their expression during bud dormancy in peach (Prunus persica). Plant Physiol Biochem. 2016;104:54-70.

43. Gatz C. From pioneers to team players: TGA transcription factors provide a molecular link between different stress pathways. Mol Plant-Microbe Interact. 2013;26(2):151-9.

44. Wang Y, Salasini BC, Khan M, et al. Clade I TGA bZIP transcription factors mediate BLADE-ON- PETIOLE-dependent regulation of development. Plant Physiol. 2019;180(6):937-51.

45. Peng Y, Sun T, Zhang Y. Perception of salicylic acid in Physcomitrella patens. Front Plant Sci. 2017:8:2145.

46. Alves MS, Dadalto SP, Gonçalves AB, et al. Plant bZIP transcription factors responsive to pathogens: a review. Int J Mol Sci. 2013;14(4):7815-28.

47. Fujita Y, Yoshida T, Yamaguchi-Shinozaki K. Pivotal role of the AREB/ABFSnRK2 pathway in ABRE-mediated transcription in response to osmotic stress in plants. Physiol Plant. 2013;147(1):15-27.

48. Wang L, Li M, Liu Z, et al. Genome-wide identification of CNGC genes in Chinese jujube (Ziziphus jujuba Mill.) and ZjCNGC2 mediated signalling cascades in response to cold stress. BMC Genomics. 2020;21(1):1-16.

49. Li H, Gao W, Xue C, et al. Genome-wide analysis of the bHLH gene family in Chinese jujube (Ziziphus jujuba mill.) and wild jujube. BMC Genomics. 2019; 20:568.

50. Hua Z, Doroodian P, Vu W. Contrasting duplication patterns reflect functional diversities of ubiquitin and ubiquitin-like protein modifiers in plants. Plant J. 2018;95(2):296-311.

51. Guo AY, Zhu QH, Chen X, et al. GSDS: a gene structure display server. Hereditas. 2007:29(8):1023-6

52. Hall BG. Building phylogenetic trees from molecular data with MEGA. Mole Biol Evol. 2013;30(5):1229-35.

53. Saitou N, Nei M. The neighbor-joining method: a new method for reconstructing phylogenetic trees. Mol Biol Evol. 1987;4(4):406-25.

54. Bu J, Zhao J, Liu M. Expression stabilities of candidate reference genes for RT-qPCR in Chinese jujube (Ziziphus jujuba Mill.) under a variety of conditions. PLoS One. 2016;11(4):e0154212.

55. Livak KJ, Schmittgen TD. Analysis of relative gene expression data using real-time quantitative PCR and the $2^{-\Delta \Lambda C T}$ method. Methods. 2001;25(4): $402-8$.

56. Xue C, Li H, Liu Z, et al. Genome-wide analysis of the WRKY gene family and their positive responses to phytoplasma invasion in Chinese jujube. BMC Genomics. 2019:20:464.

\section{Publisher's Note}

Springer Nature remains neutral with regard to jurisdictional claims in published maps and institutional affiliations.

\section{Ready to submit your research? Choose BMC and benefit from:}

- fast, convenient online submission

- thorough peer review by experienced researchers in your field

- rapid publication on acceptance

- support for research data, including large and complex data types

- gold Open Access which fosters wider collaboration and increased citations

- maximum visibility for your research: over $100 \mathrm{M}$ website views per year

At BMC, research is always in progress.

Learn more biomedcentral.com/submissions 